\title{
O Conhecimento das Gestantes acerca das Sorologias solicitadas durante o Pré-Natal
}

\author{
Bllenda Cristina Santos Ferreira ${ }^{1}$; Nívea Maria Silveira de Almeida ${ }^{2}$
}

\begin{abstract}
Resumo: O presente estudo tem por objetivo geral: avaliar o conhecimento das gestantes acerca das sorologias que são solicitadas durante os trimestres gestacionais. E como objetivos específicos: caracterizar o perfil sociodemográfico das participantes em estudo; identificar as sorologias mais conhecidas entre as gestantes; e identificar as fontes de informação sobre as sorologias. Trata-se de uma pesquisa de caráter descritivo-exploratório de abordagem quantitativa realizada na Estratégia de Saúde da Família (ESF) na cidade de Ibotirama, município do estado da Bahia, com amostra de 20 (vintes) gestantes. As informações foram obtidas por meio de um questionário. Para participar da pesquisa, as gestantes assinaram o Termo de Consentimento Livre e Esclarecido, sendo a pesquisa aprovada pelo Comitê de Ética em Pesquisa da Faculdade Independente do Nordeste. Após a coleta dos dados, os mesmos foram inseridos em uma planilha do Programa Excel e posteriormente transportados para o Programa Estatístico SPSS versão 20.0, sendo realizada análise descritiva e expostos por meio de tabelas, bem como discutidos a partir de referenciais teóricos. Os resultados permitem inferir que a avaliação dos percentuais em relação ao atendimento às gestantes reflete a qualidade do cuidado prestado. Esses dados podem ser usados para o planejamento das ações com a realização de campanhas que venham a contribuir com aumento da adesão das gestantes ao pré-natal, além de reforçar o atendimento mais qualificado.
\end{abstract}

Palavras-chave: Gestantes. Pré-Natal. Sorologias.

\section{The Knowledge of Pregnant Women about the prologue requested during Prelate}

\begin{abstract}
The objective of the present study is to evaluate the knowledge of pregnant women about the serologies that are requested during the gestational trimesters. And as specific objectives: to characterize the sociodemographic profile of the study participants; Identify the most common serologies among pregnant women; And identify sources of information on serologies. This is a descriptive-exploratory research of a quantitative approach carried out in the Family Health Strategy (ESF) in the city of Ibotirama, in the state of Bahia, with a sample of 20 pregnant women. The information was obtained through a questionnaire. To participate in the research, the pregnant women signed the Free and Informed Consent Form, and the research was approved by the Research Ethics Committee of the Faculdade Independente do Nordeste. After the data were collected, they were inserted into an Excel Program worksheet and later transported to the SPSS Statistical Program version 20.0. Descriptive analysis was carried out and the data were presented through tables, as well as discussed from theoretical references. The results allow to infer that the evaluation. These data can be used to plan the actions with the accomplishment of campaigns that will contribute to increase the adhesion of the pregnant women to prenatal.
\end{abstract}

Keywords: Pregnant women. Prenatal. Serologies.

${ }^{1}$ Graduanda em Enfermagem pela Faculdade Independente do Nordeste - FAINOR. Contato: bllenda_cris@hotmail.com;
${ }^{2}$ Mestre em Enfermagem e Saúde pelo Programa de Pós-Graduação em Enfermagem e Saúde da UESB. 


\section{Introdução}

O Sistema Único de saúde é um dos marcos históricos de maior relevância em nosso país, e uma das etapas mais importantes que podemos citar, é a da criação do Ministério da Saúde, firmando conceitos que hoje são fundamentais. A partir do momento em que a democratização foi reconhecida, foi possível uma ativa e efetiva participação popular e, desta forma, a expressão da constituição do ano de 1988, definindo, portanto a criação de um sistema com base em um atendimento universal, equitativo, com bases e preceitos legais sobre a promoção, proteção e recuperação de todo e qualquer indivíduo que procure o serviço de saúde (DOMINGUES, 2012).

O Programa de Saúde da Família (PSF) se constituiu na principal estratégia para a mudança do modelo assistencial existente, com intuito de se utilizar de novas práticas, tanto organizacionais quanto do próprio atendimento, com bases em novos conhecimentos e critérios. Esta perspectiva é totalmente positiva, pois retira o indivíduo como projeto central, e inclui sua família, ou seja, não é visto apenas os fatores individuais, mas sim a participação familiar como objeto fundamental (GUERREIRO et al., 2012).

Desta forma, é possível uma melhoria, tanto das condições assistenciais, como das relações familiares, ou seja, o serviço também atua aproximando estas pessoas. Apesar das dificuldades para sua inserção, transpor barreiras é fundamental, de forma a modificar muitos conceitos, organizações e estratégias dentro destas unidades, ou até mesmo extramuros, refletindo nas relações sociais e no trabalho (BRASIL, 2012).

O Pré-Natal é conhecido como o período que antecede o nascimento de um bebê, desde a sua pré-concepção, até o parto. Durante esse processo, são realizadas orientações, o profissional deve conhecer e tirar as dúvidas das gestantes, solicitar exames laboratoriais e procedimentos previamente estabelecidos e isto inclui explicar sobre todas estas sorologias e as suas patologias abrangentes (NUNES, 2012).

Dentro deste contexto, o enfermeiro é inserido de maneira fundamental, o qual realiza papel essencial nas ações oferecidas neste serviço, pois no pré-natal esse profissional deve prestar um cuidado delicado e sensitivo, de maneira a simplificar e ajudar na sua aproximação 
com a paciente, garantir atenção a saúde materna e fetal, realizar acompanhamento juntamente com uma equipe multidisciplinar, agindo com relação a seus medos, dúvidas e angustias relacionadas a este período, realizar o cadastramento destas gestantes, elabora planos assistenciais, realizar a consulta propriamente dita, solicitar exames, realizar atividades grupais e dá outras providências relacionadas a este estágio (FIGUEIRO FILHO et al., 2012).

Durante o Pré-Natal, são solicitados exames e procedimentos, de extremo valor e importância, pois é através deles que se é possível fazer um diagnóstico de doenças, de maneira efetiva e funcional, reduzindo os riscos maternos ou de exposição fetal neste período, podendo citar patologias como a AIDS, sífilis, dentre outras tantas, nos possibilitando intervir, preferencialmente de maneira precoce, principalmente com relação a patógenos que ultrapassem a barreira placentária e acabem atingindo o feto. Estes exames devem ser realizados no mínimo duas vezes durante toda a gestação, a saber: no primeiro e último semestre (AMARAL, 2012).

Nesta perspectiva, o presente estudo tem por objetivo geral: avaliar o conhecimento das gestantes acerca das sorologias que são solicitadas durante os trimestres gestacionais. E como objetivos específicos: caracterizar o perfil sociodemográfico das participantes em estudo; identificar as sorologias mais conhecidas entre as gestantes; e identificar as fontes de informação sobre as sorologias.

A relevância da realização de tal pesquisa se justifica na importância que tem estes exames na saúde materno-infantil e no subsídio que a pesquisa pode vir a fornecer a profissionais de saúde para melhorar a comunicação com as gestantes acerca da temática, garantindo seu direito à informação e melhorando, possivelmente, a adesão das gestantes aos protocolos de exames a serem realizados.

Informações adequadas sobre pré-natal é de fundamental importância, uma vez que permite que o cliente exerça os seus direitos, reconheça os métodos contraceptivos e faça escolhas independentes.

Assim sendo, essa pesquisa científica almeja revelar informações que possam fundamentar cada vez mais a prática da enfermagem, no que diz respeito a orientar gestantes com relação aos seus anseios, medos e qualquer incerteza no seu ciclo-gravídico. 


\section{Metodologia}

Com a finalidade de alcançar os objetivos propostos no início da pesquisa, o presente trabalho caracteriza-se por adotar a pesquisa teórico-empírica. A pesquisa teórica é "dedicada a reconstruir teorias, conceitos, ideias, ideologias, polêmicas, tendo em vista, em termos imediatos, aprimorar fundamentos teóricos" (DEMO, 2004, p. 20). Ainda segundo Demo (2004, p. 36), “o conhecimento teórico adequado acarreta rigor conceitual, análise acurada, desempenho lógico, argumentação diversificada, capacidade explicativa".

O presente estudo também é do tipo descritivo-exploratório. É descritivo, pois visa descrever as características de determinada população ou fenômeno ou o estabelecimento de relações entre variáveis. É exploratório, pois envolve levantamento bibliográfico, e questionário com pessoas que tiveram experiências práticas com o problema pesquisado (GIL, 2010).

A pesquisa referida no presente estudo foi executada na cidade de Ibotirama, município do estado da Bahia, responsável por oito Estratégias de Saúde da Família (ESF). A cidade está localizada distante aproximadamente $622,6 \mathrm{~km}$ via $\mathrm{BR}$ - 242, ocupando uma área territorial de 1.391,232 Km² apresentando uma população de 27.405 habitantes (IBGE, 2014).

A coleta de dados foi realizada na ESF Centro, responsável pelo atendimento à população adscrita deste bairro. Além dos demais profissionais que compõem a ESF, esta unidade possui uma Enfermeira que realiza os atendimentos e consultas referentes ao Pré-Natal e demais consultas de enfermagem. Estas consultas são realizadas pelo menos uma vez na semana, atendendo cerca de 16 gestantes no dia agendado. Esta unidade foi escolhida mediante a facilidade de obtenção dos dados e pela vinculação tanto com a cidade, por ser o local de origem da pesquisadora, quanto pelo vínculo com a enfermeira responsável pela unidade de saúde.

Esta coleta foi realizada mediante autorização dos responsáveis pela Unidade e Município, assim como das gestantes entrevistadas. A amostra de estudos foi representada por 20 gestantes. Para a realização desta pesquisa, foi necessária a utilização de critérios de inclusão e exclusão. Foram incluídas, portanto, as gestantes cadastradas no pré-natal da unidade, para as quais já tenha sido solicitado as sorologias. O único critério de exclusão foia recusa em participar, por qualquer motivo, e menores de idade. As informações foram obtidas por meio de um questionário. 
Esta pesquisa atendeu a Resolução 466/12 que trata da realização de pesquisas com seres humanos. Para tanto, o projeto foi submetido e aprovado pelo Comitê de Ética e Pesquisa da Faculdade Independente do Nordeste (CAAE: 66827417.1.0000.5578) e seus dados só foram coletados após aprovação do Comitê. Para participar da pesquisa, as gestantes assinaram o Termo de Consentimento Livre e Esclarecido, sendo garantido sigilo e anonimato aos participantes, atendendo os aspectos éticos de pesquisa.

Após a coleta dos dados, os mesmos foram inseridos em uma planilha do Programa Excel e posteriormente transportados para o Programa Estatístico SPSS versão 20.0, sendo realizada análise descritiva e expostos por meio de tabelas, bem como discutidos a partir de referenciais teóricos.

\section{Resultados e Discussão}

Após tratamento dos dados, os mesmos foram dispostos em tabelas a fim de facilitar sua visualização e interpretação.

$\mathrm{Na}$ Tabela 01 abaixo são apresentados os principais fatores socioeconômicos das pessoas pesquisadas. A faixa etária das mulheres variou de 18 anos a 40, sendo que a maioria tinha entre 31 a 35 anos. No que diz respeito à escolaridade, a maioria $(40,00)$ cursaram apenas o ensino fundamental completo. No que concerne à renda familiar, observa-se que a grande maioria das gestantes possui até 01 salário mínimo $(60,00)$, o que comprova uma renda inferior.

Tabela 1: Perfil Sócio-Demográfico. Ibotirama/BA, 2017

\begin{tabular}{lcc}
\hline \multicolumn{1}{c}{ Variáveis do estudo } & $\mathbf{N}$ & $\%$ \\
\hline \multicolumn{1}{c}{ Idade } & & \\
$18-25$ anos & 04 & 20,00 \\
$26-30$ anos & 05 & 25,00 \\
$31-35$ anos & 06 & 40,00 \\
$36-40$ anos & 05 & 25,00
\end{tabular}




\section{Gestações}

Primigesta

Multigesta

\section{Estado Civil}

Com companheiro

Sem companheiro

\section{Escolaridade}

Sem escolaridade

Ensino Fundamental Completo

Ensino Médio Completo

Outros

\section{Renda Mensal Familiar}

Até 01 salário mínimo

De 01 a 02 salários mínimos

Mais de 02 salários mínimos
$12 \quad 60,00$

$\begin{array}{ll}06 & 30,00 \\ 14 & 70,00\end{array}$

$12 \quad 60,00$

$08 \quad 40,00$

$\begin{array}{ll}02 & 10,00 \\ 08 & 40,00 \\ 06 & 30,00 \\ 04 & 20,00\end{array}$

$06 \quad 30,00$

$02 \quad 10,00$

\begin{tabular}{rrr}
\hline Total & 20 & 100,00 \\
\hline
\end{tabular}

Fonte: Dados coletados pela autora da pesquisa. 2017.

Esses resultados permitem inferir que as mulheres estão situadas em uma faixa etária não considerada de risco, visto que apenas 26,00 das mulheres estão acima dos 36 anos conforme apontam os estudos de Amaral (2012).

Com relação à situação conjugal, a maioria das gestantes entrevistadas $(60,00)$ tem companheiro. Esse resultado evidencia, pois, segundo as ideias de Domingues et al., (2013) uma maior estabilidade das relações conjugais o que pode facilitar o apoio do marido/companheiro no compartilhar das responsabilidades e dificuldades cotidianas. Isso é considerado um fator relevante para a pesquisa.

Observa-se, pois, por esse resultado que a baixa instrução pode favorecer o desconhecimento quanto aos programas de saúde e, por conseguinte, pode contribuir com a desinformação para o autocuidado (DOMINGUES, 2012).

Infere-se dos resultados que as gestantes vivem em condição de inferioridade econômica e social. Corroborando as ideias de Mesquita et al., (2012, p. 05) “A associação pobrezadesinformação se constitui fator de risco para a vulnerabilidade das mulheres mantendo relação direta com as limitações de suas relações pessoais". 
Pelos dados apresentados na Tabela 02 todas as mulheres entrevistadas já realizaram o exame HIV, seguido de 70,00 referente á rubéola, 50,00 sífilis, 40,00 toxoplasmose, 30,00 citomegalovírus e 10,00 HTLV.

Observa-se pelos dados da Tabela 03, apresentada abaixo, o desconhecimento de algumas sorologias, como, por exemplo, o citomegalovírus. Os que obtiveram um maior índice foi o do HIV e da rubéola que corresponderam a um percentual significativo de 100,00.

Tabela 2: Percentual dos exames realizados. Ibotirama/BA, 2017.

\begin{tabular}{lccc}
\hline & \multicolumn{1}{c}{ Variáveis do estudo } & N & $\%$ \\
\hline \multicolumn{1}{c}{ Quais dos seguintes exames você já realizou? } & 06 & 30,00 \\
Citomegalovírus & 04 & 40,00 \\
Toxoplasmose & 02 & 10,00 \\
HTLV & 20 & 100,00 \\
HIV/AIDS & 14 & 70,00 \\
Rubéola & 10 & 50,00 \\
Sífilis & Total & 20 & $100 \%$ \\
\hline
\end{tabular}

Fonte: Dados coletados pela autora da pesquisa. 2017.

Tabela 3: Percentual do conhecimento do significado dos exames. Ibotirama/BA, 2017.

\begin{tabular}{|c|c|c|}
\hline Variáveis do estudo & $\mathbf{N}$ & $\%$ \\
\hline \multicolumn{3}{|l|}{$\begin{array}{l}\text { Quais dos seguintes exames você conhece o } \\
\text { significado? }\end{array}$} \\
\hline Citomegalovírus & 00 & 00,00 \\
\hline Toxoplasmose & 02 & 10,00 \\
\hline HTLV & 06 & 30,00 \\
\hline HIV/AIDS & 20 & 100,00 \\
\hline Rubéola & 20 & 100,00 \\
\hline Sífilis & 16 & 80,00 \\
\hline Total & 20 & $100 \%$ \\
\hline
\end{tabular}

Fonte: Dados coletados pela autora da pesquisa. 2017.

De acordo com BRASIL (2012, p. 68) o Programa de Humanização no Pré-Natal e Nascimento, é critério fundamental para o acompanhamento pré-natal a solicitação dos 
seguintes exames: Grupo sangüíneo e fator Rh (quando não realizado anteriormente); Sorologia para sífilis (VDRL); Urina tipo I; Hemoglobina e hematócrito (Hb/Ht); Glicemia de jejum; Teste anti-HIV com aconselhamento pré-teste e consentimento da mulher; Sorologia para hepatite B (HBsAg), se disponível; Sorologia para toxoplasmose, se disponível; Colpocitologia oncótica, quando indicada.

A assistência ao pré-natal consiste num conjunto de atividades e procedimentos realizados para assegurar a boa evolução da gestação e tem como objetivo principal acolher a mulher desde o início de sua gravidez, que se caracteriza por um período de mudanças físicas e emocionais, que cada gestante vive de maneira distinta (ANVERSA, 2012).

Desse modo, as consulta de pré-natal são extremamente importantes para a saúde da mulher como também para o feto. De acordo com as ideias de Amaral (2012) constituem como os principais objetivos das consultas de pré-natal: avaliar o estado de saúde da mãe e do feto, determinar a idade gestacional, iniciar um plano de cuidados do pré-natal, identificar as possíveis complicações da gestação, dentre outros fatores.

A autora ainda lembra que as primeiras consultas do pré-natal devem ser iniciadas o quanto antes para que possam ser feitos os exames necessários à realização de um adequado acompanhamento da gestante e do bebê. Além disso, quando realizados no início da gravidez, os referidos exames podem apontar alguma doença ou disfunção que pode ser tratada e corrigida a tempo, desde que diagnosticadas precocemente. Daí a importância de se começar o pré-natal o mais cedo possível (AMARAL, 2012).

Confirmando tal visão, Nunes $(2012$, p.16) afirma que "a realização do pré-natal é essencial a fim de prevenir e detectar precocemente patologias tanto maternas quanto fetais". Para a autora, a troca de informações e de relatos de experiência entre as gestantes e os profissionais de saúde constitui a melhor forma de promover a compreensão do processo de gestação.

A Tabela 04 abaixo apresenta a fonte de informação para o conhecimento dessas sorologias, que pelos dados apresentados, o profissional de saúde $(60,00)$ tem uma efetiva participação para a instrução do autocuidado das gestantes. 
Tabela 4: Fonte de informação das orientações de saúde. Ibotirama/BA, 2017.

\begin{tabular}{lccc}
\hline \multicolumn{1}{c}{ Variáveis do estudo } & N & $\%$ \\
\hline $\begin{array}{l}\text { Qual foi a fonte de informação para conhecimento do } \\
\text { significado dessas sorologias? }\end{array}$ & & \\
Família & 02 & 10,00 \\
Mídia & 06 & 30,00 \\
Profissional de Saúde & 12 & 60,00 \\
\hline & Total & 20 & $100 \%$ \\
\hline
\end{tabular}

Fonte: Dados coletados pela autora da pesquisa. 2017.

Neste sentido, a assistência de enfermagem deve buscar estabelecer uma comunicação clara com as gestantes, promovendo o intercâmbio de informações e experiências, uma vez que a gestante tem o direito de ser informada acerca do seu estado de saúde, bem como do seu bebê e de participar das decisões que envolvem a sua vida (NUNES, 2012).

Cabe ressaltar que a atuação do enfermeiro está regulamentada pela Lei ${ }^{\circ}$. 7.498, de 25 de junho de 1986, que dispõe sobre o exercício da enfermagem. Além disso, o Decreto $\mathrm{n}^{\circ}$ 94.406, de 08/06/1987, Art. $8^{\circ}$, incube ao profissional dessa área, privativamente, dentre várias atribuições, a prestação de assistência de enfermagem à gestante e a participação na elaboração e na operacionalização do sistema de referência nos diferentes níveis de atenção à saúde (AMARAL, 2012).

Sabe-se que o enfermeiro, para conseguir prestar uma assistência adequada às gestantes, deve procurar conhecê-las bem, especialmente no que se refere à natureza de seus problemas e dos recursos que ela possui para resolvê-los, levando em consideração seus hábitos, crenças e valores, tendo em conta que a assistência à saúde não pode ser vista de maneira dissociada de outros aspectos, como os sociais, religiosos, políticos e econômicos (SENA, 2014).

Assim, pode-se notar que os profissionais da saúde precisam transcender o foco biomédico na atenção ao pré-natal, passando a considerar os aspectos multifatoriais que influenciam a vida da gestante em todo seu contexto ambiental (DOMINGUES, 2012). Dessa forma, é importante que o enfermeiro se disponha a ouvir com atenção e paciência as gestantes para que possa auxiliá-la durante todo o período gestacional, especialmente quando se considera que tal período, além de ser marcado por mudanças no aspecto físico da mulher, também pode ser marcado por alterações no aspecto comportamental da mesma. 
Contudo, corroborando os estudos de Guerreiro et al., (2012) percebe-se, ainda, que mesmo que as políticas de atendimento à mulher tenham avançado ainda persistem algumas "lacunas" de falta de conhecimento, o que requer dos profissionais de saúde um maior cuidado.

A atuação dos enfermeiros no cuidado, gerenciamento de recursos humanos e materiais, liderança, planejamento da assistência, capacitação da equipe de enfermagem desperta novas abordagens associando ideias de novos espaços de atuação do enfermeiro (SANTOS et al., 2013).

Portanto, atividades de educação em saúde são recursos que permitem a aproximação entre profissionais e receptores do cuidado além de contribuírem para o oferecimento de assistência humanizada. Neste sentido, é interessante que se efetuem trocas de experiências entre as mulheres e os profissionais de saúde - ações educativas, pois facilitam a compreensão do processo gestacional. É através da educação que as mudanças sociais podem ocorrer, sendo cada encontro com o outro transformado num momento de troca, crescimento e resolução (LIMA; MELO; FERREIRA, 2012).

\section{Considerações Finais}

Para garantir uma gestação e um parto com saúde, toda gestante precisa fazer o prénatal. Quanto mais cedo procurar um serviço de saúde, maiores as chances de o bebê nascer saudável. É durante o pré-natal que a mulher faz os exames necessários para saber se ela e seu filho estão bem.

A avaliação dos percentuais em relação ao atendimento às gestantes reflete a qualidade do cuidado prestado. Esses dados podem ser usados para o planejamento das ações com a realização de campanhas que venham a contribuir com aumento da adesão das gestantes ao prénatal, além de reforçar o atendimento mais qualificado.

Assim, ao iniciar uma assistência ao pré-natal deve se manter um atendimento qualificado e humanizado, tendo em vista ações de promoção a saúde para melhor compreensão da mulher durante este período. Para que a enfermagem faça uma assistência completa e adequada a profissional deve estar em harmonia com a área que está atuando e aberta para o acolhimento a gestante e sua família. O papel importante da enfermagem durante o pré-natal 
são as orientações feita a mulher, proporcionando a retirada de dúvidas e uma troca de experiência com outras mulheres gestantes.

Fazem-se necessários estudos qualitativos subsequentes para melhor caracterização do serviço dos profissionais e da população adscrita ao ESF Centro no município de Ibotirama. Vale destacar, ainda, que os objetivos deste estudo foram alcançados.

Para melhor entendimento das gestantes, o ideal é que criassem mais campanhas voltadas para conscientização da prevenção de doenças sexualmente transmissíveis antes de iniciar sua vida sexual para que assim as metas pudessem ser compridas e mais mulheres fossem imunizadas, assim diminuiriam os riscos de adquirir um vírus que causariam sérios danos a saúde desse grupo populacional no futuro.

\section{Referências}

AMARAL, Aline. Nível de Conhecimento das Gestantes Atendidas em uma UBS no Município de Livramento de Nossa Senhora - BA sobre a Importância do Pré-natal. 55f. Monografia (Graduação em Enfermagem) - 2012. Faculdade de Tecnologia e Ciências, Vitória da Conquista/BA.

ANVERSA ETR. Qualidade do processo da assistência pré- natal: unidades básicas de saúde e unidades de Estratégia Saúde da Família em município no Sul do Brasil. Cad Saúde Pública,2012.

BRASIL. Programa de Humanização no Pré-Natal e Nascimento. Brasília (DF): Ministério da Saúde; 2012.

DEMO, Pedro. Pesquisa e Construção do conhecimento. Rio de Janeiro: Templo Brasileiro, 2004

DOMINGUES, R. M. S. M.et al. Sífilis congênita: evento sentinela da qualidade da assistência pré-natal. Rev. Saúde Pública, São Paulo, v. 47, n. 1, Feb. 2013.

DOMINGUES HDL. Avaliação da adequação da assistência pré-natal na rede SUS do município do Rio de Janeiro, Brasil. Cad Saúde Pública. 2012;28(3):425-37.

FIGUEIRÓ-FILHO, E. A. et al. Sífilis e Gestação: Estudo Comparativo de Dois Períodos (2006 e 2011) em População de Puérperas.DST- J bras Doenças Sex Transm, Rio de Janeiro, v.24, n. $1: 32-37,2012$. 
GIL, Antônio Carlos. Métodos e técnicas de pesquisa social. São Paulo: Atlas, 2010.

GUERREIRO, E, et al., O cuidado pré - natal na atenção básica de saúde sob o olhar de gestantes e enfermeiros, Rev. Min. Enferm.;v16, n(3), P.5: 315-323, jul./set., 2012.

IBGE. Dados estatísticos da cidade de Ibotirama/BA. Disponível em: www.ibge.gov.br. Acesso em: 09/05/2017.

LIMA, A.F, MELO, A. M. A. A, FERREIRA, M. A. Pré-natal: um desafio para as gestantes nas unidades de saúde da família no município de Serra Talhada-PE. Rev. Enfermagem Saúde coletiva em debate, V.2 N.1, p.31-40, dez, 2012

MESQUITA, K. O. et al. Análise dos casos de sífilis congênita em Sobral, Ceará: contribuições para assistência pré- natal. DST. J. bras. doenças sex. transm. Rio de Janeiro, v.24, n. 1, 2012.

NUNES, Helaine Aparecida de Faria. Assistência ao Pré-natal de Baixo Risco na Estratégia de Saúde da Família e seus Desafios: uma revisão de literatura. 2012.

SANTOS. J, et al.Práticas de enfermeiros na gerencia do cuidado em enfermagem e saúde revisão integrativa.Rev Bras Enferm,66(2):257-63,mar-abr,2013.

SENA, I. V. A. Qualidade da Atenção Pré-Natal na Estratégia Saúde da Família: Revisão de Literatura. 2014.

Como citar este artigo (Formato ABNT):

FERREIRA, Bllenda C.S.; ALMEIDA, Nívea M.S. O Conhecimento das Gestantes acerca das Sorologias solicitadas durante o Pré-Natal. Id on Line Revista Multidisciplinar e de Psicologia, Abril de 2017, vol.11, n.35, p.155-167. ISSN: 1981-1179.

Recebido: 20.05.2017

Aceito: 20.05 .2017 


\section{Anexo}

\section{QUESTIONÁRIO}

\section{Dados sociodemográficos}

Faixa Etária:

( ) 18 a 25 anos ( ) 25 a $30 \operatorname{anos}($ ) 31 a 35 anos ( ) 35 a 40 anos

Gestações:

( ) Primigesta ( ) Multigesta

Estado Civil:

( ) Com Companheiro ( ) Sem companheiro

Escolaridade:

( ) Sem escolaridade ( ) Ensino Fundamental Completo ( ) Ensino Médio Completo ( ) Outros

Renda Mensal:

( ) Até 01 salário mínimo ( ) De 01 a 02 salários mínimos ( ) Acima de 02 salários mínimos

\section{PARTE II}

Quais dos seguintes exames você já realizou:

( ) Citomegalovírus ( ) Toxoplasmose ( ) HTLV ( ) HIV/AIDS ( ) Rubéola ( ) Sifilis

Quais dos seguintes exames você conhece o significado:

( ) Citomegalovírus ( ) Toxoplasmose ( ) HTLV ( ) HIV/AIDS ( ) Rubéola ( ) Sifilis

Qual foi a fonte de informação para conhecimento do significado dessas sorologias?

( ) Família ( ) Mídia ( ) Profissional de Saúde 